\title{
Aproximación a la gestión económica integral de las obras por procesos productivos: elaboración del modelo COP de control de costes de construcción
}

\section{Approach to the comprehensive economic management of building works by production processes: Elaboration of the COP model for building cost control}

\author{
M. V. Montes $^{(*)}$, M. E. Ponce ${ }^{(*)}$, R. M. Falcón ${ }^{(*)}$, A. Ramírez-de-Arellano ${ }^{(*)}$
}

\section{RESUMEN}

La profunda crisis económica sufrida recientemente en España ha puesto de manifiesto la vulnerabilidad del Sector de la Construcción y la necesidad de desarrollar nuevas herramientas que incrementen su eficacia. Centrándonos en el aspecto económico de las obras, un acuciante problema es la importante desviación existente entre presupuesto y coste real en numerosas intervenciones. En aras de paliar este desajuste, se propone la definición de un modelo COP de control de costes basado en procesos productivos que extrapola, a la fase de seguimiento y control económico de las obras, los planteamientos desarrollados en el modelo POP de presupuestación de obras por procesos. Así, la aplicación combinada de los modelos POP y COP en las obras de edificación proporciona una gestión económica eficaz e integral, que se adapta fielmente a su planificación y desarrollo, posibilitando la precisa identificación de desviaciones y la adopción de medidas correctoras para reajustar los errores detectados.

Palabras clave: Control de costes, gestión económica integral, procesos, planificación, obra de edificación, economía de la construcción.

\section{ABSTRACT}

The deep economic crisis recently undergone in Spain has highlighted the vulnerability of the Construction Industry and the necessity of the development of new tools to increase its efficiency. Focusing on the economic dimension of building works, a pressing and growing problem is the significant deviation identified among estimates and real building costs. In order to solve this failure, a model for building cost control based on production processes is proposed, the COP model. This model extrapolates the approach of the model for estimating building construction costs by processes, the POP model, to the phase of monitoring and economic control of building construction works. Therefore, the combined implementation of both models, POP and COP, provides an efficient and comprehensive economic management of building works by accurately adjusting to their planning and development and facilitating the effective identification of deviations and adoption of corrective measures to settle the mistakes detected.

Keywords: Cost control, comprehensive economic management, processes, planning, building works, construction economics.

(*) Universidad de Sevilla - E. T. S. Ingeniería de Edificación (España).

Persona de contacto/Corresponding author: toya@us.es (M. V. Montes)

ORCID: http://orcid.org/oooo-0003-0533-6294 (M. V. Montes); http://orcid.org/oooo-0oo1-7112-5722 (M. E. Ponce);

http://orcid.org/oooo-0002-6474-7301 (R. M. Falcón); http://orcid.org/oooo-0001-7041-7395 (A. Ramírez-de-Arellano)

Cómo citar este artículo/Citation: Montes, M. V., Ponce, M. E., Falcón R. M., Ramírez-de-Arellano, A. (2017). Aproximación a la gestión económica integral de las obras por procesos productivos: elaboración del modelo cop de control de costes de construcción. Informes de la Construcción, 69(548): e230, doi: http://dx.doi.org/10.3989/ic.16.121.

Copyright: (C) 2017 CSIC. Licencia / License: Salvo indicación contraria, todos los contenidos de la edición electrónica de Informes de la Construcción se distribuyen bajo una licencia de uso y distribución Creative Commons Attribution License (CC BY) Spain 3.o. 


\section{INTRODUCCIÓN}

La diferencia entre los ingresos generados y gastos soportados constituye el parámetro primordial a la hora de determinar el grado de eficacia del conjunto de agentes que actúan en todo proceso económico (1). En caso de que, por determinados factores circunstanciales, un aumento de ingresos no sea factible, la manera natural de optimizar dicha eficacia es mediante un mayor control y seguimiento de los gastos en que incurre el proceso. Estos factores circunstanciales a los que se ha hecho mención se hacen evidentes en momentos de crisis económica global como la sufrida desde comienzos de 2008.

En España, uno de los sectores económicos que más ha sufrido la mencionada crisis económica es el de la Construcción, cuya vulnerabilidad ante los distintos movimientos de mercado se ha puesto claramente de manifiesto en los últimos años. En relación a la pérdida de eficacia de los agentes que intervienen en toda ejecución de obra, esta circunstancia se debe fundamentalmente a la importante desviación entre presupuesto y coste real que usualmente se vislumbra en gran parte de las obras que son ejecutadas (2). Si bien en los últimos años se han estudiado distintas formas de solventar la creciente pérdida de competitividad y eficacia económica de dicho sector (3-4), no ha sido hasta muy recientemente que se ha puesto el foco de atención en la posibilidad de ajustar y optimizar el modelo detallado de presupuestación por unidades de obra (5), el modelo más utilizado a nivel nacional para la estimación de los costes esperados de las obras de edificación proyectadas mediante la consideración de costes directos e indirectos de ejecución. Como alternativa a dicho modelo y basado en las reconocidas metodologías ABC (Activity-Based Costing) (6-7) y PBC (Process-Based Costing) (8-10), Montes et al. (11-12) han introducido recientemente el modelo POP de presupuestación de obras por procesos, el cual incorpora todos los costes vinculados con el centro de producción de las obras, a excepción de los impuestos, por vía directa. Para ello la obra de edificación se descompone en las actividades vinculadas a la producción de los trabajos identificados a partir de su correspondiente planificación, constituyendo los denominados procesos de ejecución; y éstos a su vez se descomponen en los recursos necesarios para su desarrollo, los denominados procesos básicos. De este modo el presupuesto por procesos proporciona una estimación transparente y personalizada para cada obra de todos sus costes, ajustada a la forma en que realmente se construye.

El presente artículo resuelve en particular uno de los problemas abiertos que fueron planteados por Montes et al. (12). Concretamente, el desarrollo de un modelo integral de gestión de obras de edificación basado en procesos productivos que permita la mejora de la eficacia económica durante la fase de construcción de las obras de edificación. Basado en el modelo POP, se introduce en particular el denominado modelo COP de control de costes por procesos productivos, con el que se pretende comprobar que los costes en que se incurren durante el proceso constructivo se corresponden con los previstos en el estudio inicial, con la intención de que, si no fuera así, se pueda proceder a analizar las desviaciones y tomar las medidas correctoras necesarias.

\section{CONTEXTUALIZACIÓN DEL MODELO COP}

Atendiendo al modelo detallado de presupuestación de unidad de obra introducido por Ramírez-de-Arellano en 1998
(5), cualquier ejecución de obra puede considerarse, desde el punto de vista económico, como un todo indivisible del denominado sistema global de edificación. Recientemente, a nivel temporal, Basabe del Castillo (13) ha identificado tres subsistemas principales en el ciclo de vida de dicho sistema: S1 (Preconstrucción), S2 (Construcción) y S3 (Postconstrucción).

Al comenzar la actividad del subsistema $\mathrm{S} 2$ ya se conocen el programa de necesidades y el volumen de inversión disponible. No obstante, en la práctica y dependiendo de cada caso concreto, distintos mecanismos de regulación y control permiten considerar permeable la frontera entre los subsistemas S1 y S2. Esto posibilita corregir perturbaciones internas y externas que amenacen el equilibrio global del sistema. Así, por ejemplo, una frontera inicial entre dichos subsistemas puede venir dada por:

- La asignación de un solar a un proyecto del que se ha definido un programa de necesidades y al que se ha dotado de los recursos económicos necesarios.

- La realización de los trabajos de demolición necesarios para iniciar con libertad el nuevo edificio o las tareas de transformación profunda del edificio actual.

Por su parte, la frontera entre los subsistemas S2 y S3 es simple y claramente identificable: la recepción definitiva del edificio, una vez que se han terminado las obras y se ha liquidado el contrato con la empresa constructora.

El modelo COP de control de costes por procesos productivos que introducimos en el presente artículo constituye un subsistema englobado dentro de la citada fase S2. Con vistas a facilitar su posterior análisis, identificamos en la Figura 1 la estructura jerarquizada en la que se basan los niveles de desarrollo que se detallan a continuación.

\subsection{Nivel No: Sistema Global Edificación}

Se corresponde con el sistema en su totalidad. En él se sitúa la finalidad principal del modelo en relación a la edificación, que pretende realizar un seguimiento y control económico de la actividad de la empresa constructora adjudicataria durante la fase de ejecución de los procesos productivos, debiéndose cumplir para ello los siguientes principios:

1. El constructor debe saber qué ejecutará y cuánto cobrará.

2. El constructor debe diseñar los procesos y estimar sus costes (modelo POP).

3. Todo recurso se imputará en el proceso usado.

\subsection{Nivel N1: Subsistema S2}

Se establecen como premisas que el promotor disponga de un solar o edificio a transformar en el que se han realizado las demoliciones necesarias; que se haya realizado el correspondiente proyecto y que se disponga de la financiación y de los permisos administrativos necesarios. En este nivel se concentra el núcleo de actividades de la obra, materializándose una sucesión de fases, hasta tener el proceso completo, y así conseguir desde el punto de vista del modelo los siguientes objetivos:

1. Construir el edificio según proyecto.

2. Cumplir los plazos contractuales. 


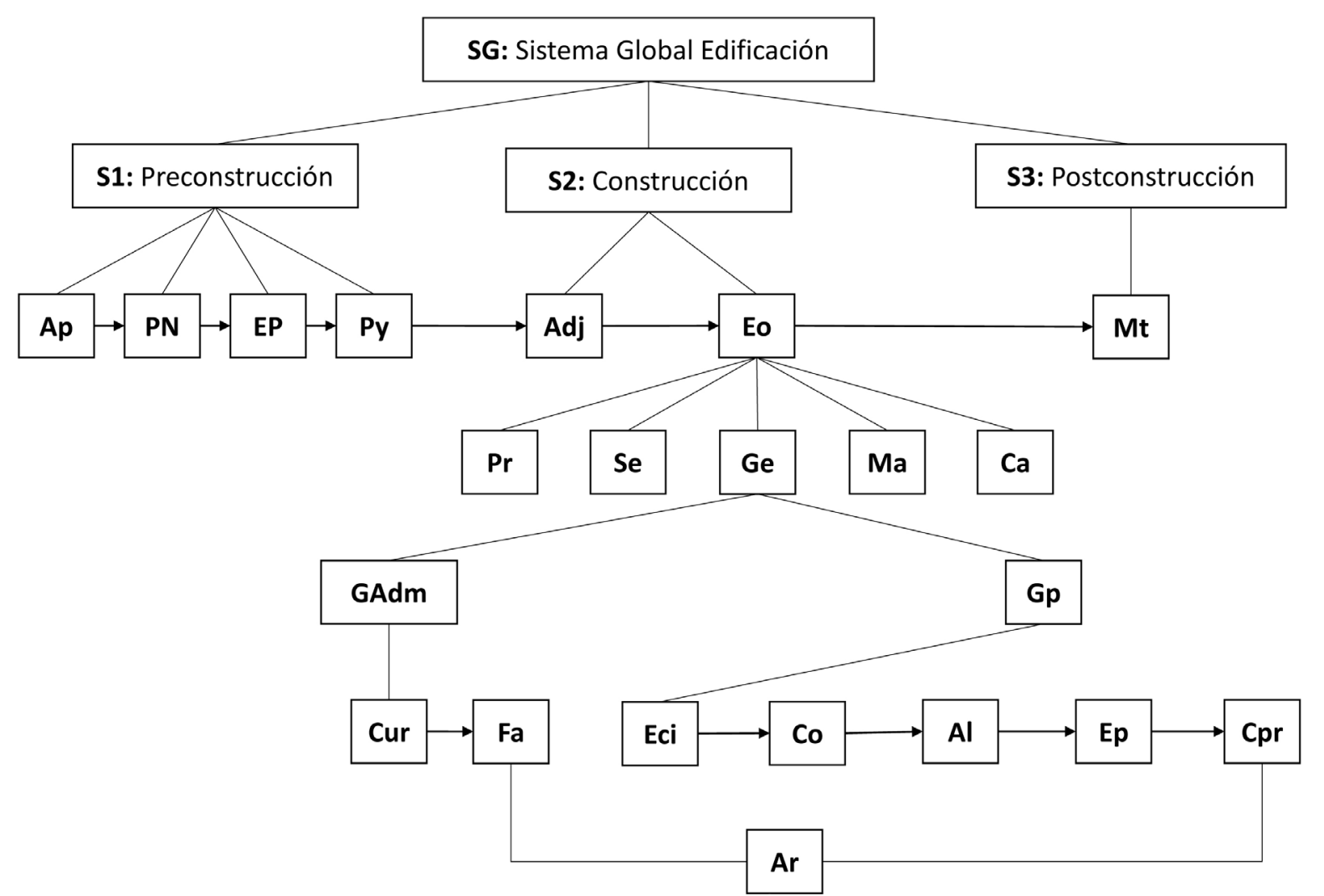

Nivel 0

Nivel 1

Nivel 2

Nivel 3

Nivel 4

Figura 1. Sistema Global basado en el modelo de Ramírez-de-Arellano. (Ap: Actuaciones preliminares; PN: Programa de necesidades; EP: Estudios previos ; Py: Proyecto; Adj: Adjudicación; Eo: Ejecución de obra; Mt: Programa de mantenimiento; Pr: Producción; Se: Seguridad y salud; Ge: Gestión económica ; Ma: Gestión medioambiental ; Ca: Gestión de la calidad; GAdm: Gestión administrativa; Gp: Gestión de la producción ; Cur: Cuantificación de recursos; Fa: Facturación de recursos; Eci: Estimación inicial; Co: Compras; Al: Almacén; Ep: Ejecución de procesos; Cpr: Cuantificación de la producción; Ar: Análisis de resultados de control).

3. Respetar los parámetros de calidad, seguridad y medioambientales establecidos y superar todas las pruebas de servicio.

4. Conseguir que no existan desviaciones negativas entre la estimación de costes inicial y el coste real.

5. Liquidar y acreditar la finalización de obra mediante la firma del acta de recepción por los agentes implicados en el proceso.

\subsection{Nivel N2: División del subsistema S2 en dos etapas}

En este nivel se desarrollan todas las actividades relacionadas con el control de costes por procesos a través de las siguientes dos etapas:

- Etapa de Adjudicación (Adj). Disponiendo de un proyecto de ejecución y de las autorizaciones necesarias y conociéndose con detalle el volumen de inversión, se siguen las pautas de la Ley de Contratos del Sector Público (14) o los criterios establecidos por un promotor privado, con vistas a elegir la empresa constructora adjudicataria y definir el presupuesto de adjudicación.

- Etapa de Ejecución de obra (Eo). Disponiendo de un solar sobre el que puede ser construido una edificación y habiéndose elegido la empresa constructora adjudicataria, en esta fase es donde tiene lugar la actividad productiva en sí misma, desarrollándose todos aquellos procesos que darán lugar al producto final. Como objetivo se plantean:

1. Recibir de conformidad el edificio construido.

2. Realizar la liquidación de la obra ejecutada.
Las actividades desarrolladas en ambas etapas darán respuestas de forma sucesiva a los objetivos del modelo planteados por medio de los siguientes hitos:

1. Estructurar y regularizar la política de gestión económica de la empresa.

2. Disponer de una planificación de costes (POP de ejecución).

3. Disponer de un registro de las operaciones y actividades realizadas.

4. Informar de la situación en que se encuentra el sistema.

5. Dotar al sistema de un soporte para el análisis y toma de decisiones.

\subsection{Nivel N3: División de la etapa Eo en cinco subsistemas}

En este nivel se desarrollan todas las actividades asociadas directamente a la etapa de ejecución de obra atendiendo a los siguientes subsistemas:

- Subsistema de producción (Pr). Disponiendo de un proyecto de adjudicación y de un plazo contractual para la terminación de las obras objeto del mismo, se formaliza la documentación contractual y se firma el acta de inicio de obra. Como objetivos se plantean:

1. Diseñar los procesos productivos y el mapa de procesos de ejecución descompuesto en procesos básicos.

2. Ejecutar los procesos productivos previstos hasta la terminación de la obra, recibiendo de conformidad el edificio construido. 
- Subsistema de seguridad y salud (Se). Disponiendo de un estudio básico de seguridad, se realiza el plan de seguridad de la obra y se firma el acta de aprobación por el coordinador de seguridad. Como objetivos se plantean:

1. Nombrar el recurso preventivo.

2. Realizar la seguridad en la ejecución de los procesos productivos hasta la terminación de la obra.

- Subsistema de gestión económica (Ge). Disponiendo de un proyecto de adjudicación, con unos costes estimados por el departamento de estudios y de un plazo contractual para la terminación de las obras objeto del proyecto, se formaliza la documentación contractual y se firma el acta de inicio de obra. Se plantean como objetivos:

1. Controlar los costes de producción de forma periódica hasta la terminación de la obra.

2. Recibir la conformidad del edificio construido.

3. Obtener la información económica final.

- Subsistema de gestión medioambiental (Ma). Disponiendo de un proyecto con un estudio de gestión de residuos y habiéndose aprobado el plan de gestión de residuos elaborado por la empresa constructora, se realiza el seguimiento y control de la gestión de residuos hasta su entrega en planta de tratamiento o vertedero autorizado con la emisión de certificado de entrega, hasta la recepción de la obra de conformidad.

- Subsistema de gestión de la calidad (Ca). Disponiendo de un proyecto con un presupuesto de adjudicación y de un plazo contractual para la terminación de las obras objeto del proyecto, se elabora el plan de aseguramiento de la calidad, y se realiza el seguimiento y control del mismo hasta la recepción de la obra de conformidad.

\subsection{Nivel N4: División del subsistema Ge en dos áreas}

Se plantea en este nivel la división del sistema de gestión económica en dos áreas diferenciadas, pero complementarias a la vez:

- Área de gestión administrativa (GAdm). Disponiendo de los datos reales de la producción realizada a origen, se contabilizan y fiscalizan los mismos. Como objetivo se plantea obtener la información económica de todo el proceso productivo.

- Área de gestión de la producción (Gp). Disponiendo de la estimación de costes y programación del departamento de estudios, en base a ella el equipo de producción realiza la estimación de costes inicial y se diseñan los procesos y procedimientos de ejecución. Como objetivo se plantea desarrollar las actividades tanto estratégicas como de toma de datos para dar apoyo al núcleo de operaciones responsable de la ejecución de los procesos, cumpliendo para ello los subsistemas que de ella cuelgan en el nivel N5 a describir a continuación.

\subsection{Nivel N5: División de las áreas del nivel N4 en subsistemas}

En relación al área de administración, una vez obtenidos los datos de la producción en el periodo en cuestión, tanto de ejecución como de consumo de recursos, se elaboran las correspondientes facturas proforma de los recursos adquiridos, codificadas de forma conveniente. Se plantea entonces la subdivisión de dicho área en los siguientes dos subsistemas:
- Subsistema de cuantificación de recursos (Cur). Se trasladan las facturas proformas y los albaranes codificados al departamento de administración. Se plantea registrar e imputar los datos de los consumos de recursos, ya codificados, al proceso que lo ha utilizado, además de obtener la información económica del periodo. Se plantean como objetivos:

1. Elaborar una estructura contable codificada según el mapa de procesos.

2. Identificar los centros de coste.

3. Imputar los costes codificados en los centros identificados.

4. Obtener los resultados de la contabilidad de costes del periodo.

5. Obtener informes de costes.

- Subsistema facturación de recursos (Fa). Se remiten las facturas proforma a proveedores y subcontratistas para su posterior facturación y se trasladan a producción para su verificación y autorización. Se plantean como objetivos:

1. Dar entrada a la factura en administración.

2. Conformar la factura por los departamentos implicados.

3. Imputar la factura real contra la factura proforma.

Por su parte, en relación al área de gestión de la producción se plantea su división en cinco subsistemas:

- Subsistema estimación inicial (Eci). Partiendo del proyecto existente de estimación de costes, de la programación del departamento de estudios, del presupuesto de adjudicación y del contrato de ejecución de obra, el equipo de producción pide ofertas de los recursos en los mercados y elabora la estimación de costes según la estructura y clasificación sistemática del modelo de procesos productivos y realiza la programación de los trabajos en base a los procedimientos de ejecución que realmente se utilizarán, obteniendo la estimación inicial de costes mediante el modelo POP de ejecución de obra. Este modelo servirá de referencia durante la ejecución de los procesos productivos, siendo aceptado por la dirección de producción. Los objetivos de esta etapa son:

1. Estudiar y analizar el proyecto.

2. Elaborar el mapa de procesos.

3. Determinar los procedimientos de ejecución de los procesos.

4. Organizar la estructura interna de referencia (EIR).

5. Estimar la cantidad de recursos necesarios en cada proceso.

6. Estimar el coste de los recursos necesarios.

7. Obtener una planificación de coste global de referencia (POPe).

- Subsistema de compras (Co). Una vez dispuesto el modelo POP de ejecución de la obra y conocidos los resultados conseguidos en el subsistema Cur, se debe dar respuesta a la planificación del coste global definido con un presupuesto de costes que no supere el volumen de gasto aprobado. Partiendo de esa frontera, el departamento de compras selecciona distintas empresas para la solicitud de ofertas y adjudicación de los trabajos, en base a las solicitudes emitidas por el departamento de producción. Los objetivos de esta etapa son:

1. Recibir las solicitudes de compras de producción.

2. Seleccionar empresas homologadas.

3. Emitir las peticiones de oferta.

4. Recibir y elaborar un comparativo de ofertas.

5. Adjudicar y contratar la oferta más adecuada.

6. Emitir los pedidos de los recursos. 
- Subsistema almacén (Al). Una vez realizadas las compras en base a las solicitudes emitidas por el departamento de producción, se busca recibir los recursos en obra registrándose para ello la entrada de los mismos, así como las salidas correspondientes, estas últimas con indicación del proceso de destino. Los objetivos planteados son:

1. Custodiar y gestionar la distribución a los procesos de los recursos adquiridos en el subsistema anterior.

2. Dar entrada a los recursos adquiridos asignándole el código correspondiente del modelo de gestión por procesos.

3. Realizar y mantener relación valorada por códigos de los recursos almacenados.

4. Dar salida, mediante albarán, del recurso codificado con indicación del código del proceso de destino según el mapa de procesos.

5. Trasladar, en los periodos fijados, los albaranes de salida al departamento de administración.

- Subsistema de ejecución de procesos (Ep). Una vez dispuestos los recursos necesarios se puede comenzar la ejecución de obra con vistas a terminar el proceso con la conformidad de la propiedad. Los objetivos planteados en este momento son:

1. Materializar la ejecución de los procesos.

2. Ejecutar los procesos según el mapa diseñado.

3. Optimizar el consumo de recursos.

4. Cumplir los plazos previstos.

5. Ejecutarlos cumpliendo los objetivos de calidad.

6. Gestionar los residuos generados.

- Subsistema de cuantificación de la producción (Cpr). Habiéndose iniciado las obras y transcurrido el periodo establecido por la empresa para la obtención de datos, este subsistema sienta uno de los dos pilares fundamentales del modelo COP: la cuantificación de la producción y de los recursos utilizados en la misma. Se trata de una toma de datos in situ de lo realmente ejecutado, según el mapa de procesos y el POP de ejecución elaborado, con vistas a cuantificar los procesos ejecutados a origen. Esta cuantificación se realizará en los periodos establecidos. Los objetivos asignados a esta etapa son:

1. Cuantificar los procesos ejecutados.

2. Obtener el coste estimado de la producción en el periodo según el POP de ejecución.

3. Cuantificar y codificar los recursos utilizados.

4. Asignar cada recurso codificado al proceso asignado.

5. Elaborar facturas proforma codificadas de subcontratistas y remitirlas.

6. Trasladar el informe de producción, coste previsto y consumos de recursos (coste real) a administración.

\subsection{Nivel N6: Análisis de resultados del control (Ar)}

En este nivel encuentra su sentido último el modelo desarrollado, ya que de la información obtenida en los niveles anteriores se podrá determinar la situación de la obra y tomar decisiones enfocadas a subsanar posibles desviaciones o a mejorar los procesos diseñados. Para ello se parte de los costes reales ocasionados en el periodo y registrados en contabilidad y de los costes estimados de la producción en el periodo registrados en el POP de ejecución. Habiéndose imputado a los procesos los costes a origen del periodo, y realizada la comparación de lo estimado y lo real obtenido, se analizan las desviaciones obtenidas profundizando en las posibles causas de las mismas. Los objetivos de este nivel son:
1. Realizar un comparativo de costes, real versus previsto.

2. Encontrar los puntos discordantes.

3. Analizar los diseños de los procesos con discrepancias.

4. Proponer medidas correctoras o rediseñar los procesos.

5. Establecer plazos para la implantación y seguimiento de dichas medidas correctoras.

Como fase final del modelo COP se plantea la toma de decisiones para la cual se dispone del análisis de las desviaciones detectadas con una propuesta de las posibles causas y estudio de las mismas. Se proponen tanto medidas correctoras tendentes a corregir las causas de la desviación, como estrategias para anular o minimizar los efectos de las desviaciones detectadas.

\section{ESTRUCTURA TEMPORAL DEL MODELO COP}

En esta sección se analiza la estructura temporal del modelo COP, identificando para ello los subprocesos que le dan forma y explicando la evolución de los escenarios temporales que componen el subsistema de gestión económica. Dicha estructura temporal es ilustrada en la Figura 2, donde el análisis diacrónico de la evolución del sistema global utilizado como imagen de referencia se corresponde a la sección longitudinal de la trayectoria del sistema, con hitos representados por los momentos de tiempo to a t10, los cuales son utilizados para situar las secciones transversales que ubicarán los escenarios sincrónicos. La secuencia de estos últimos ayudará a describir la evolución de los dos grandes procesos (GAdm y Gp) utilizados para explicar el modelo. Analizamos a continuación los distintos escenarios e instantes de evolución de dicha estructura temporal.

\subsection{Evolución [to - t1]: Adjudicación}

En este periodo, el promotor, ya sea público o privado, ha recibido las ofertas de las empresas que han participado en el proceso, adjudicando la obra a la empresa que se ajuste a los criterios fijados.

\subsection{Escenario t1: Ejecución de obra}

Es el origen de la evolución del ciclo de vida del modelo. En este escenario se sitúan los actores principales desde el punto de vista de la gestión económica:

- Promotor que realiza la inversión.

- Empresa constructora adjudicataria de la obra.

- Dirección facultativa como controlador del proceso.

\subsection{Evolución [t1 - t2]}

Una vez determinada la empresa adjudicataria se formaliza el contrato de ejecución de obra, entre promotor y constructor, en base al proyecto de ejecución existente y la oferta presentada por el constructor. El promotor designa al equipo de dirección de obra y se procede a la firma del acta de inicio de obra.

\subsection{Escenario t2: Gestión económica}

Terminada la primera evolución, en este tramo aparecen los actores propios de la empresa adjudicataria, que se suman a los mencionados en el periodo anterior. Este escenario tiene como origen la firma del acta de inicio de obra y tendrá como documento director del mismo el contrato de ejecución de obra con la oferta de adjudicación basada en el estudio de costes del departamento de estudios. 


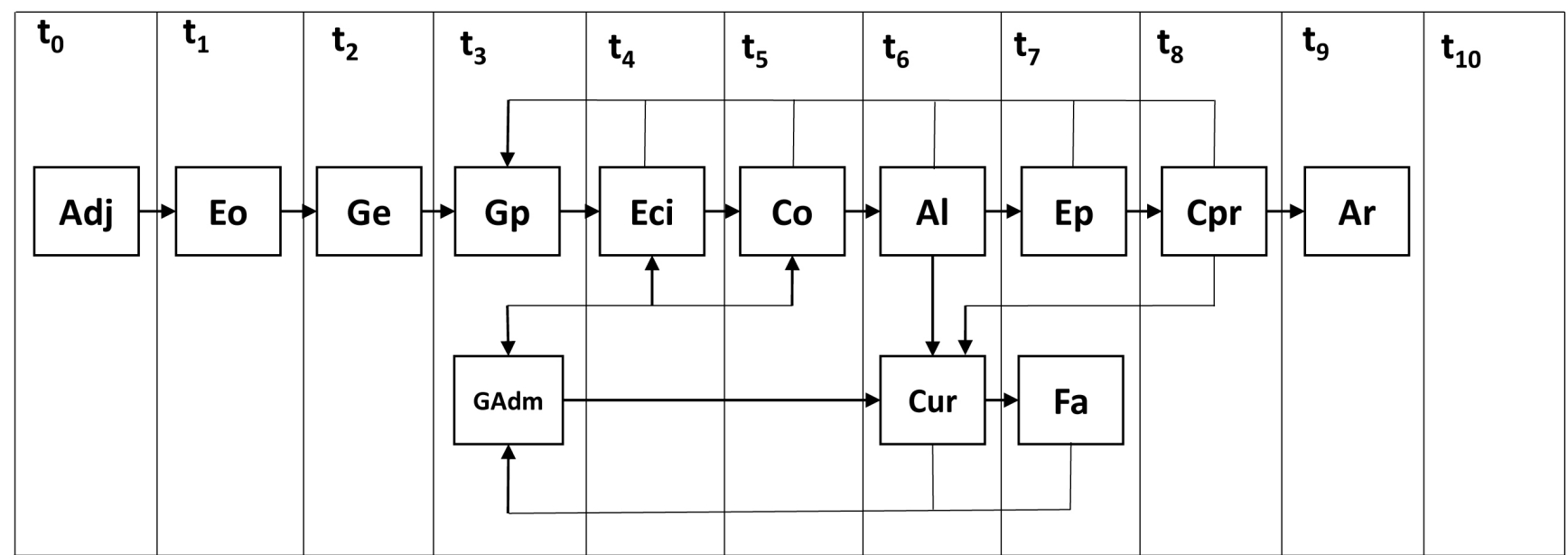

Figura 2. Estructura temporal del modelo. (Adj: Adjudicación; Eo: Ejecución de obra; Ge: Gestión económica; Gp: Gestión de la producción; Eci: Estimación inicial; Co: Compras; Al: Almacén; Ep: Ejecución de procesos; Cpr: Cuantificación de la producción; Ar: Análisis de resultados de control; GAdm: Gestión administrativa; Fa: Facturación de recursos; Cur: Cuantificación de recursos).

\subsection{Evolución [t2 - t3]}

El intervalo temporal [t2 - t3] es pieza clave del modelo COP. Aquí se debe elegir el equipo que gestionará y ejecutará los procesos productivos de la obra, tanto a nivel de producción como de administración, basándose en los documentos anteriores.

\subsection{Escenario t3}

Es este escenario encontramos ya los dos grandes procesos que caracterizan el modelo: la gestión económica de la producción de procesos (Gp) y la gestión administrativa (GAdm).

\subsection{Evolución [t3 - t4]}

Se designan por parte de la dirección de la empresa los equipos responsables de cada proceso y se les hace entrega de los documentos contractuales iniciales.

\subsection{Escenario t4: Estimación de costes inicial}

Como consecuencia del punto anterior, es en este momento en el que se establece la estimación de costes inicial haciendo uso del modelo POP de ejecución.

\subsection{Evolución [t4 - t5]}

En este intervalo temporal se desarrolla el estudio de costes inicial (POP de ejecución) por el equipo de producción designado, debiéndose a la vez diseñar los procesos y su mapa correspondiente, decidir los procedimientos de ejecución y la estructura interna de referencia. Todo ello con una programación de los procesos diseñados para su aprobación por la dirección de producción y posterior traslado al departamento de administración para su inclusión en el sistema de gestión (ERP) de la empresa.

\subsection{Escenario t5: Gestión de compras de recursos}

Aprobado el POP de ejecución, se tiene un marco de referencia de costes, que se mantendrá a lo largo de la obra y servirá de base para la adquisición de los recursos necesarios.

\subsection{Evolución [t5 - t6]}

El objetivo asignado a este tramo es la compra y contratación en el mercado de los recursos necesarios para la ejecución de los procesos. Dicha compra se realiza de forma coordinada con el responsable del correspondiente proceso de ejecución. Concretamente, mediante las solicitudes de compra realizadas por el equipo de producción y teniendo como referencia los costes de los recursos estimados en el POP de ejecución, el departamento de compras realiza la petición de ofertas de los recursos a las empresas colaboradoras.

\subsection{Escenario t6: Gestión de almacén y cuantificación de recursos}

Llegados a este punto ya se ha firmado el acta de inicio de obra, se han constituido los equipos de trabajo y se ha realizado la apertura del centro de trabajo. Se puede iniciar por tanto el proceso productivo, para lo cual es necesario disponer en el centro de producción de los recursos necesarios para la ejecución de los procesos productivos. Se habilita para ello en dicho centro un espacio para almacenar y gestionar estos recursos.

\subsection{Evolución [t6 - t7]}

En este tramo se produce la entrada en obra de los recursos necesarios para la ejecución de los procesos y se registra la entrada de los mismos llevando un control de existencias. Asimismo se registra la salida de los recursos hacia los procesos de ejecución, todo ello utilizando la codificación del modelo POP $(12,15)$. El departamento de almacén remite los albaranes de entrada ya codificados de los proveedores para su registro y posterior unión a la factura del proveedor. $\mathrm{Al}$ final del periodo se remite al departamento de contabilidad y al de producción la relación valorada de existencias.

\subsection{Escenario t7: Ejecución de los procesos y facturas de proveedores}

El objetivo de este escenario es la materialización de los procesos productivos ejecutados de forma secuenciada y ordenada según el POP de ejecución. Para ello se debe contar en el centro de producción con los recursos e implantación necesarios. 


\subsection{Evolución [t7 - t8]}

En este periodo se ejecutan los procesos productivos según la planificación aprobada. Dado que ya han entrado recursos en el centro de producción se produce la llegada de las facturas que las empresas colaboradoras emiten por sus servicios, las cuales son registradas por el departamento de contabilidad.

\subsection{Escenario t8: Cuantificación de los procesos y certificación de los procesos}

En este escenario el equipo de producción realiza la cuantificación de los procesos ejecutados tomando datos in situ de los mismos.

\subsection{Evolución [t8 - t9]}

Este es uno de los momentos claves del modelo ya que en base a la cuantificación realizada por medio de la toma de datos in situ se obtiene la información necesaria para explicar los objetivos y finalidades del proceso. Esta información se comparte con el departamento de contabilidad para obtener los informes necesarios. De igual forma sirve para emitir la certificación del periodo a la dirección facultativa.

\subsection{Escenario t9: Análisis de resultados}

Este es el escenario principal del modelo pues confluyen los datos obtenidos de la cuantificación. Una vez transcurrido el periodo de tiempo establecido por la empresa constructora para realizar los controles económicos periódicos se procede al estudio y análisis de los resultados obtenidos por comparación entre la programación y estimación de costes inicial, como imagen de referencia, con los resultados obtenidos de la toma de datos reales.

\subsection{Evolución [t9 - t1o]}

A partir de la información obtenida en las etapas anteriores se puede determinar la situación económica-temporal de la obra y tomar decisiones enfocadas a controlar y subsanar posibles desviaciones o a mejorar los procesos diseñados. Para ello se debe analizar si la producción ejecutada tanto a origen como en el periodo en cuestión, así como el coste de dicha producción, es mayor, igual o menor que la prevista. Los dos primeros indicadores orientan sobre posibles retrasos o adelantos en el plazo de ejecución de la obra, mientras que el tercero refleja si el resultado económico evoluciona respecto a las expectativas creadas en la estimación inicial de costes. Para llevar a cabo estos aspectos se realizan las siguientes tareas:

a) Convocatoria de reunión de seguimiento mensual

Finalizada la toma de datos y formalizada la contabilidad del periodo, los datos obtenidos han sido reflejados en el formato de producción y trasladados a los órganos de dirección de la empresa. Dicho órgano de dirección convoca a los responsables de obras el día diez del mes siguiente al periodo cerrado, para el estudio de la evolución de la obra y de los resultados económicos de la misma.

b) Realización de comparativo de costes, real versus previsto

En el formato de producción, se tienen reflejados los costes previstos de la producción ejecutada a origen y el coste real de dicha producción, reflejándose las diferencias.

\section{c) Localización y análisis de desviaciones}

Para la localización y análisis de discordancias se estudia si la producción ejecutada a origen es mayor, igual o menor que la prevista. Para dar respuesta a esta cuestión hemos de apoyarnos en la programación de los procesos incluida en la planificación inicial aprobada, la cual se encuentra vigente. En dicha programación nos fijamos en el mes correspondiente al periodo en cuestión y obtenemos la producción prevista a origen y en el mes. Realizando la diferencia entre la producción real ejecutada y la prevista, obtenemos los siguientes indicadores de la evolución de la obra en cuanto a plazo.

$$
\mathrm{DNP}=\mathrm{IPE}-\mathrm{IPP}
$$$$
\mathrm{DP} \%=\mathrm{DNP} \times 100 / \mathrm{IPP}
$$

Siendo:

- DNP: Desviación neta en producción.

- IPE: Importe de producción ejecutada a origen.

- IPP: Importe de producción prevista a origen.

- DP\%: Desviación porcentual en producción.

Si ambos indicadores [1] y [2] se anulan, no existen desviaciones. Si ambos son positivos, existe un avance en la ejecución de los procesos y por tanto se está acortando el plazo de ejecución previsto. Finalmente, si ambos son negativos, se ha ejecutado menos obra de la prevista, por lo que habrá que analizar las causas de dicho retraso y proponer medidas correctoras.

El análisis de la producción ejecutada en el periodo bajo consideración se realiza de forma análoga a lo recién expuesto. Por su parte, independientemente de que la cantidad de procesos ejecutados se corresponda o no con la cantidad prevista en la programación de los procesos, se ha de controlar si los costes reales ocasionados son mayores o menores que los previstos. Para ello nos apoyamos en el formato de producción, donde se ha reflejado el precio de la producción a origen y en el periodo. El coste previsto para dicha producción según la estimación inicial de costes y el coste real según la contabilidad viene determinado por los siguientes indicadores (por diferencias y por porcentajes, respectivamente):

$$
\begin{gathered}
\mathrm{DNC}=\mathrm{C}-\mathrm{CPP} \\
\mathrm{DC} \%=\mathrm{DNC} \times 100 / \mathrm{CPP}
\end{gathered}
$$

Siendo:

- DNC: Desviación neta en costes.

- C: Coste real contabilizado por la administración.

- CPP: Coste previsto de la producción.

- DC\%: Desviación porcentual en costes.

Si ambos indicadores [3] y [4] se anulan, entonces estamos cumpliendo los objetivos marcados. Si son negativos, existe un menor coste respecto a la estimación de costes inicial. Finalmente, si son positivos, existe un mayor coste respecto a la estimación de costes inicial, debiendo realizar un análisis en los distintos niveles del presupuesto para poder profundizar en las posibles causas de las desviaciones. Además, dado que no es lo mismo, por ejemplo, una desviación de un 10\% en el coste complejo de un proceso básico que suponga el $2 \%$ de su proceso de ejecución que esa misma desviación de un 10\% 
en un proceso básico que suponga el $40 \%$ de su proceso de ejecución, es necesario determinar cuándo las mencionadas desviaciones tienen la suficiente entidad como para que requieran la intervención en los procesos afectados. Para ello introducimos aquí el concepto de peso del proceso:

$$
\mathrm{PPB}=\mathrm{CCPB} / \mathrm{CCPE} \times 100
$$

Siendo:

- PPB: Peso porcentual específico del proceso básico.

- CCPB: Coste complejo del proceso básico.

- CCPE: Coste complejo del proceso de ejecución.

Combinando el porcentaje de desviación en costes con el peso del proceso, obtenemos un indicador adimensional que considera la desviación y la importancia en cuanto a participación del proceso básico en el proceso de ejecución, debiendo actuar sobre aquellos procesos cuyo indicador sea superior a una cifra establecida.

$$
\mathrm{D}=\mathrm{DC} \% \times \mathrm{PPB}
$$

Siendo:

- D: Indicador de desviación.

- DC\%: Desviación porcentual en costes.

- PPB: Peso porcentual específico del proceso básico.

En cuanto al valor límite de este indicador a partir del cual debemos actuar sobre el diseño del proceso, proponemos dos criterios:

- Establecer un valor fijo para toda la cartera de obras vinculada a la empresa constructora bajo consideración, dependiendo del riesgo de posibles pérdidas económicas que esté dispuesta a asumir la misma. Pudiendo estar contemplado por tanto de forma constante en la planificación estratégica de la empresa, se trata de un valor subjetivo cuya función consiste en encender la luz de alarma una vez se haya consumido la parte del beneficio neto que se haya determinado como tope máximo. Así, por ejemplo, tomando como referencia una desviación del 2,5\% en el coste complejo de un proceso cuyo peso porcentual específico fuese un $20 \%$, el valor del indicador a considerar sería 50.

- Establecer un valor calculado para cada obra por separado en base a los beneficios previstos en la estimación de costes inicial. De este modo, la empresa establece de antemano el valor límite porcentual del beneficio neto de la obra en cuestión que estaría dispuesta a perder. Dicho valor constituye de hecho el incremento de coste máximo establecido por la empresa. La desviación máxima por porcentaje en costes referenciada al beneficio neto vendría dada entonces por el indicador:

$$
\mathrm{DM} \%=\mathrm{BN} \times \mathrm{Lím} \times 100 / \mathrm{CPP}
$$

Siendo:

- DM\%: Desviación porcentual máxima de los beneficios.

- BN: Beneficio neto del proceso básico.

- Lím: Valor límite del beneficio neto que se está dispuesto a perder.

- CPP: Coste previsto de la producción.
Dado que el modelo de control de costes por procesos permite un diseño particularizado para cada obra, proponemos aplicar este indicador de desviación calculado a medida y para cada proceso básico, mediante el cálculo de su indicador de desviación máxima para un proceso referenciado a su margen neto según la siguiente ecuación:

$$
\mathrm{DM}=\mathrm{DM} \% \times(\mathrm{CCPB} \times 100 / \mathrm{CCPE})
$$

Siendo:

- DM: Desviación máxima del proceso básico.

- DM\%: Desviación porcentual máxima de los beneficios.

- CCPB: Coste complejo del proceso básico.

- CCPE: Coste complejo del proceso de ejecución.

El punto de aviso en cuanto al porcentaje de beneficio neto a consumir lo determinarán los órganos de dirección para cada obra. Para ello en el impreso de estimación de costes inicial se añadirán las columnas correspondientes al beneficio neto del proceso, el porcentaje de desviación máxima y el indicador límite de desviación.

Finalmente, un último indicador habrá de medir el beneficio de la empresa constructora. Para ello se ha de constatar si el resultado económico final del periodo a origen se corresponde con el previsto, dado que ese es el fin último. De ese modo, basándonos en el coste previsto de la producción, el coste real de la misma y el precio de la producción a origen comprobaremos si:

$$
\begin{gathered}
\mathrm{IPE}-\mathrm{CPP}<\mathrm{IPE}-\mathrm{C} \\
\mathrm{BP}<\mathrm{BO}
\end{gathered}
$$

Siendo:

- IPE: Importe de la producción ejecutada.

- CPP: Coste previsto de la producción.

- C: Coste real contabilizado por la administración.

- BP: Beneficio antes de impuestos previsto.

- BO: Beneficio antes de impuestos obtenidos según la contabilidad.

d) Proposición de medidas correctoras y establecimiento de plazos para su implantación y seguimiento

En caso de que se identifiquen retrasos en el desarrollo de los procesos hay que proceder a analizar sus causas. A continuación se relacionan las más frecuentes en obras de edificación, así como una serie de posibles medidas correctoras que pueden paliarlas:

- Falta de definición del proyecto: Solicitud de detalles a la dirección facultativa.

- Diseño erróneo de los procesos: Optimización de procedimientos mediante el empleo de medios auxiliares adecuados.

- Falta de materiales en obra: Optimización y seguimiento de la planificación de suministros marcando hitos en el programa de obras.

- Falta de rendimiento de empresas colaboradoras: Penalización o sustitución de las empresas colaboradoras de bajo rendimiento.

- Paralización por inclemencias meteorológicas: Recuperación del tiempo perdido mediante el aumento de los recursos asignados al proceso, tales como por ejemplo incremento del número de equipos o doblado de los turnos de trabajo. 
Asimismo, si se detectan incrementos de costes en procesos, entonces es necesario de nuevo analizar sus causas para poder adoptar la medida correctora oportuna. Detallamos las más frecuentes, así como las medidas correctoras que se pueden adoptar para minimizar su impacto:

- Mayor cuantificación real de recursos: Revisión de la exactitud de dicha estimación y la cantidad enviada de cada recurso, comprobando el material devuelto a almacén; mejora de la transmisión de información entre almacén y producción; comprobación de pérdidas por roturas en la ejecución del proceso; y reestudio del diseño del proceso y del procedimiento de ejecución.

- Costes de no calidad: Penalización a las empresas colaboradoras que ejecuten defectuosamente los procesos.

- Cambios en el proyecto que afectan al diseño o introducen nuevos procesos: Rediseño de los procesos y negociación de nuevos precios con la empresa promotora.

Detectadas las desviaciones y propuestas las medidas correctoras, se propone un plazo para la implantación de dichas medidas. Transcurrido este plazo se procede a verificar la implantación y la eficacia de dicha acción, emitiéndose el informe favorable o no favorable de la implantación de dicha acción correctora. Dado el carácter dinámico de la obra, la puesta en marcha de estas acciones correctoras debe ser de inmediato para evitar que siga avanzando la desviación.

e) Elaboración del informe final del periodo

De los resultados económicos obtenidos y del análisis del mismo se elabora el informe final del periodo, donde se indican:

- En relación al precio de producción: código y nombre de la obra, tipo de obra, zona de la delegación, nombre del cliente, fecha del presupuesto o contrato, fecha del periodo de cierre, estado de la obra (viva o cancelada), fecha del acta de replanteo, fecha final según contrato, fecha de terminación prevista por el equipo de producción, expedientes de la obra (indicando su estado y volumen económico previsto), certificaciones aprobadas emitidas, certificaciones cobradas, obra ejecutada a origen, obra pendiente de ejecutar, volumen de producción previsto a final de obra.

- En relación al coste de la producción: Coste real contabilizado de los procesos a origen, costes exógenos aplicados, coste pendiente según la planificación aprobada, coste total previsto a final de obra según los datos anteriores.

- En relación al resultado del periodo:

$$
\begin{gathered}
\mathrm{BP}=\mathrm{IPO}-\mathrm{C}-\mathrm{CE} \\
\mathrm{Bp}=\mathrm{Ip}-\mathrm{Cp}-\mathrm{CEp} \\
\mathrm{B}=\mathrm{IPE}-\mathrm{CE}-\mathrm{C}-\mathrm{Cp}
\end{gathered}
$$

Siendo:

- BP: Beneficio antes de impuestos previsto.

- IPO: Importe de la producción del periodo a origen.

- C: Coste real contabilizado por la administración.

- CE: Costes exógenos. Estos costes son:

- Tasas de estructura: Es la suma de las tasas de central y delegación.

- Tasas de delegación: Representa el coste de la delegación repercutido sobre todas las obras de esta, se aplica porcentualmente a la producción y lo fija anualmente la dirección de la empresa.
- Tasas de central: Representa el coste de la central de la empresa repercutido sobre todas las obras de esta, se aplica porcentualmente a la producción y lo fija anualmente la dirección.

- Bp: Beneficio pendiente antes de impuestos.

- Ip: Importe de producción pendiente.

- Cp: Coste de producción pendiente.

- CEp: Costes exógenos pendientes.

- B: Beneficio total antes de impuestos, referente al resultado previsto a final de obra según los datos del cierre.

- IPE: Importe de la producción ejecutada.

Asimismo se adjunta un listado de las acciones correctoras propuestas con indicación del plazo máximo para su implantación y puesta en marcha. Este formato cumplimentado se aporta en el cierre del mes siguiente para seguimiento de las acciones correctoras por parte de los órganos de dirección.

f) Repetición del ciclo según los periodos establecidos hasta el final del proceso productivo

Este proceso se produce reiteradamente hasta el final de la obra, obteniéndose los resultados periódicos del proceso productivo según los periodos establecidos, constituyendo una visión global de la evolución de la obra.

\section{ESTUDIO DE CASO}

Para ilustrar el modelo COP exponemos a continuación parte de una aplicación del mismo que tuvo lugar durante el primer mes de ejecución de una obra real de viviendas de protección oficial. El ejemplo en cuestión se enmarca dentro de la fase final de la estructura temporal del modelo, concretamente dentro de los periodos t8, t9 y t10, al ser los más representativos del mismo.

\section{Hipótesis de partida}

La empresa promotora inició un proceso de licitación a tanto alzado para las fases de movimiento de tierras, cimentación y estructura. Una vez identificados los recursos necesarios se elaboró el mapa de procesos de ejecución desagregados en básicos, sentando las bases de cuantificación de procesos. En este sentido, la Tabla 1 muestra algunos de los procesos que se establecieron referentes a la ejecución de un forjado.

\section{Fase de ejecución}

Dispuestos los recursos necesarios y comprobados los replanteos, dio inicio la ejecución de procesos, estableciéndose los ciclos de producción y controles económicos mensuales de cuantificación in situ de los recursos consumidos en el periodo a origen. Se obtuvo así el coste previsto para la producción ejecutada, marco de referencia a comparar con los costes reales. Como ejemplo, véase la Tabla 2, donde se cuantifican los procesos detallados en la Tabla 1 durante el primer mes de nuestra obra. Se ha obtenido una producción a origen de $55.335,24 €$ con un coste previsto de 54.224,71 $€$, siendo el real contabilizado de 56.476,03 $€$.

\section{Fase de gestión administrativa}

En esta fase final se determina la situación económico-temporal de la obra y se toman decisiones enfocadas a subsanar posibles desviaciones o a mejorar los procesos diseñados. En 
Tabla 1. Identificación de procesos básicos.

\begin{tabular}{|c|c|c|}
\hline Código & $\mathbf{u}$ & Proceso \\
\hline 1030 & $\mathrm{u}$ & Entramados horizontales e inclinados \\
\hline 103005 & $\mathrm{u}$ & $\begin{array}{l}\text { Forjados de hormigón con encofrado } \\
\text { continuo }\end{array}$ \\
\hline 1030050010 & $\mathrm{u}$ & $\begin{array}{l}\text { Ejecución de forjado de planta primera } \\
\text { bloque } 3\end{array}$ \\
\hline *1005050100 & $\mathrm{m}^{2}$ & $\begin{array}{l}\text { Aligeramiento con bloque aligerante } \\
\text { forjado reticular }\end{array}$ \\
\hline${ }^{*} 1005150005$ & $\mathrm{~m}^{3}$ & Hormigón HA-25/B/20/IIa, suministrado \\
\hline *1005900005 & $\mathrm{u}$ & Base plástica embebida en el forjado \\
\hline *6010400005 & $\mathrm{u}$ & $\begin{array}{l}\text { Ensayo resistencia a compresión de } \\
\text { hormigón }\end{array}$ \\
\hline *6010400010 & $\mathrm{u}$ & Ensayo de consistencia de hormigón \\
\hline${ }^{*} 7010200005$ & $\mathrm{~m}^{3}$ & Bombeo de hormigón \\
\hline *7010700005 & $\mathrm{m}^{2}$ & $\begin{array}{l}\text { Subcontrato MO estructura reticular } \\
\text { I/Mat. complem. }\end{array}$ \\
\hline${ }^{*} 7010700010$ & $\mathrm{~kg}$ & Subcontrato de acero ferrallado y colocado \\
\hline *7010700015 & $\mathrm{m}^{2}$ & $\begin{array}{l}\text { Mallazo electrosoldado ME B } 500 \mathrm{~S} \\
15 \times 15 \times 5 .\end{array}$ \\
\hline
\end{tabular}

la obra que nos ocupa, el departamento de administración procedió a los siguientes análisis de los resultados obtenidos según la información contable y la producción del periodo:

\section{Análisis 1. Comparación de producción ejecutada respecto a la prevista}

Se realizó la diferencia entre la producción real ejecutada y la prevista a partir de los indicadores [1] y [2] acerca de la desviación neta y porcentual en producción:

- $\mathrm{DNP}=55 \cdot 335,24 €-58.831,92 €=-3.496,68 €$.

- $\mathrm{DP} \%=-3.496,68 \times 100 / 58.831,92=-5,94 \%$.

Se había ejecutado un 5,94\% menos que la producción prevista en la programación por un importe de $-3.496,68 €$. Esto reflejó una desviación negativa en cuanto a la cantidad de obra ejecutada, lo que aumentaba de forma desfavorable el plazo previsto inicialmente para el proceso en cuestión.

Ante el retraso detectado en el proceso se analizaron las posibles causas del mismo, identificándose las inclemencias meteorológicas como su origen. Como medida correctora se adoptó la nueva planificación de los procesos siguientes y la asignación de un mayor número de recursos para recuperar el tiempo perdido, contemplándose incluso la idea de trabajar horas extraordinarias para encauzar el desarrollo de la obra al plazo inicialmente previsto.

\section{Análisis 2. Comparación del coste de producción ejecutada respecto al previsto}

Se utilizaron los indicadores [3] y [4] acerca de la desviación neta y porcentual en costes:

- $\mathrm{DNC}=56.476,03 €-54.224,71 €=2.251,32 €$.

- $\mathrm{DCo} \%=2.251,32 \times 100 / 54.224,71=4,15 \%$.

Se observa un incremento de costes de 2.251,32 $€$, equivalente a un $4,15 \%$ de incremento medio, motivado por un mayor consumo de recursos en los procesos. Como medida correctora se adoptó realizar una comprobación de pérdidas por ro-
Tabla 2. Cuantificación de los recursos asociados a los procesos básicos.

\begin{tabular}{|l|r|r|r|r|}
\hline \multicolumn{1}{|c|}{ Código } & \multicolumn{1}{c|}{ IPE } & \multicolumn{1}{c|}{ CPP } & \multicolumn{1}{c|}{ IPP } & \multicolumn{1}{c|}{ C } \\
\hline${ }^{*} 1005050100$ & $4 \cdot 500,48$ & $4.410,47$ & $4.631,59$ & $4 \cdot 400,01$ \\
\hline${ }^{*} 1005150005$ & $13.568,42$ & $13.297,05$ & $14.006,98$ & $13 \cdot 318,59$ \\
\hline${ }^{*} 1005900005$ & 15,34 & 15,032 & 19,44 & 12,96 \\
\hline${ }^{*} 6010400005$ & 112,51 & 110,25 & 120,00 & 114,00 \\
\hline${ }^{*} 6010400010$ & 4,60 & 0,00 & 0,00 & 3,80 \\
\hline${ }^{*} 7010200005$ & 971,34 & 951,34 & $1.146,88$ & $1.144,37$ \\
\hline${ }^{*} 7010700005$ & $15 \cdot 479,40$ & $15.169,81$ & $16.255,20$ & $15 \cdot 438,64$ \\
\hline${ }^{*} 7010700010$ & $19 \cdot 533,61$ & $19.142,94$ & $21.427,24$ & $20.904,62$ \\
\hline${ }^{*} 7010700015$ & $1.147,54$ & $1.127,82$ & $1.228,59$ & $1.140,84$ \\
\hline Total (1030050010) & $55 \cdot 335,24$ & $54.224,71$ & $58.831,92$ & $56.476,03$ \\
\hline
\end{tabular}

IPE: Importe de producción ejecutada a origen; CPP: Coste previsto de la producción; IPP: Importe de producción prevista a origen; C: Coste real contabilizado.

turas en la ejecución del proceso y reestudiar tanto el diseño del proceso como el procedimiento de ejecución.

\section{Análisis 3. Estudio de los indicadores de desviación}

De forma complementaria a los dos anteriores análisis se realizó un tercer análisis acerca de los datos obtenidos de los indicadores de desviación referentes a los distintos recursos integrantes en el periodo bajo consideración. Este análisis es conveniente realizarlo siempre para cada proceso básico de forma independiente puesto que, en ocasiones, una mala gestión de un proceso básico concreto puede haber sido cubierta por una buena gestión en otros y enmascarar el resultado y, recíprocamente, una buena gestión de un proceso básico puede pasar desapercibida si el resto de componentes no han sido adecuadamente gestionados.

En nuestro estudio de caso, todos los procesos básicos han tenido un comportamiento proporcional, salvo en el caso del proceso básico *6010400010, u Ensayo de consistencia de hormigón (véanse Tablas 1 y 2), que ha sido incorporado posteriormente durante la ejecución de la obra, como una mejora encaminada a subsanar una carencia del proyecto.

Detectadas las desviaciones y propuestas las medidas correctoras, se propuso un plazo de diez días para la implantación de dichas medidas. Transcurrido dicho plazo se verificó su implantación y la eficacia de dicha acción correctora, emitiéndose un informe favorable por parte del jefe de obra que constataba la recuperación del tiempo perdido y la evolución favorable de la obra conforme a la planificación inicialmente prevista.

Complementariamente, el jefe de obra elaboró la certificación del periodo y la remitió a la dirección facultativa en los términos establecidos en el contrato de ejecución de obra, para obtener el visto bueno de la misma y posteriormente dio traslado al departamento de administración que cursaría la factura a la empresa promotora y obtendría su documento de cobro.

\section{CONCLUSIONES}

En el presente artículo se ha presentado el modelo COP de costes por procesos productivos como una herramienta de gestión y control de costes y análisis de resultados que proporciona una información más fiable y acorde con el proceso productivo que la generada tradicionalmente por el modelo por unidades de obra, 
facilitando el análisis de la información del estado económico de la obra y una toma de decisiones más acertada y eficiente.

El modelo propuesto se caracteriza por controlar todo proceso de ejecución a partir de su descomposición y clasificación en sus componentes más básicas. Una conveniente codificación de las mismas posibilita la trazabilidad pormenorizada de los flujos de cada uno de los recursos utilizados. En particular, permite la elaboración de paquetes de compras de recursos ya codificados y la gestión de los mismos en almacén, identificando correctamente para ello las salidas hacia los procesos de ejecución y su imputación en la estructura contable. La completa documentación de este flujo de recursos hace que dicha imputación se realice con mayor precisión que en el modelo de unidades de obra, generando una información fiable, que facilita la toma de decisiones y el establecimiento de futuras estrategias por los órganos de dirección de la empresa constructora.

Los siguientes aspectos del modelo COP presentado han sido igualmente desarrollados a lo largo del presente artículo:

- La creación de una estructura contable a nivel de usuario, idéntica a la estructura del mapa de procesos, que proporciona una mayor exactitud en las imputaciones de los costes de los recursos.
- Una estructura de documentos estandarizada, con indicación de su secuencia de utilización, la cual es susceptible de su implantación en los ERP de las empresas constructoras.

- Unos criterios de análisis de desviaciones, planteando a su vez las posibles causas y medidas correctoras a adoptar.

- Una serie de indicadores límites de desviaciones que avisan de la necesidad de actuar sobre el diseño del proceso que lo haya sobrepasado, permitiendo actuar a tiempo sobre la desviación.

De este modo, la aplicación combinada de los modelos POP y COP en las obras de edificación proporciona una gestión económica eficaz e integral, que se adapta fielmente a su planificación y desarrollo, posibilitando la precisa estimación de los costes esperados de las obras proyectadas, la identificación de desviaciones durante el transcurso de la ejecución de las mismas y la adopción de medidas correctoras para reajustar los errores detectados.

\section{Cita en género femenino}

Las referencias a personas o colectivos figuran en el presente artículo en género masculino como género gramatical no marcado. Cuando proceda, será válida la cita en género femenino a dichas referencias.

\section{REFERENCIAS}

(1) Farrell M. J. (1957). The Measurement of Productive Efficiency. Journal of the Royal Statistical Society, Series A, 120: 253-281.

(2) Bustos, O. (2014). Factores latentes de desviación de presupuestos en proyectos de Arquitectura. Tesis Doctoral. Valencia: Universitat Politècnica de València.

(3) García-Erviti F., Armengot-Paradinas J., Ramírez-Pacheco, G. (2015). El análisis del coste del ciclo de vida como herramienta para la evaluación económica de la edificación sostenible. Estado de la cuestión. Informes de la Construcción, 67(537): e056, doi: http://dx.doi.org/10.3989/ic.12.119.

(4) Montes M. V., Monterde D., Villoria P. (2011). Approach to the Use of Global Indicators for the Assessment of the Environmental Level of Construction Products. The Open Construction and Building Technology Journal, 5(2): 141-148, doi: http://dx.doi.org/10.2174/1874836801105010141.

(5) Ramírez-de-Arellano, A. (1998). Presupuestación de obras. Sevilla: Secretariado de Publicaciones de la Universidad de Sevilla.

(6) Woo K. (2001). Activity-based costing and its application to lean construction. En: 9th Annual Conference of the International Group for Lean Construction. Singapur: National University of Singapore.

(7) Yuan, F. (2011). Applying activity-based costing approach for construction logistics cost analysis. Construction Innovation, 11(3): 259-281, doi: http://dx.doi.org/10.1108/14714171111149007.

(8) Gyoh, L. E. (1999). Design-Management and Planning for Photovoltaic Cladding Systems within the UK Construction Industry. Tesis Doctoral. Sheffield: The University of Sheffield.

(9) Pulaski, M. H. (2005). The alignment of sustainability and constructability: A continuous value enhancement process. Tesis Doctoral. State College: The Pennsylvania State University.

(10) Nguyen, H. V. (2010). Process-Based Cost Modeling to Support Target Value Design. Tesis Doctoral. Berkeley: University of California.

(11) Montes, M. V., Falcón, R. M., Ramírez-de-Arellano, A. (2014). Estimating Building Construction Costs by Production Processes. The Open Construction and Building Technology Journal, 8: 171-181, doi: http://dx.doi.org/10.2174/18748 36801408010171.

(12) Montes, M. V., Falcón, R. M., Ramírez-de-Arellano, A. (2016). Estimating Building Construction Costs: Analysis of the Process-based Estimate Model (POP Model). Revista Ingeniería de la Construcción, 31(1): 17-25, doi: http://dx.doi. org/10.4067/So718-50732016000100002.

(13) Basabe del Castillo, J. B. (2012). Costes e Ingresos Globales en Edificación. Trabajo Final del Máster Universitario en Gestión Integral en Edificación. Sevilla: Universidad de Sevilla.

(14) Cortes Generales (2011). Real Decreto Legislativo 3/2011, de 14 de noviembre, por el que se aprueba el texto refundido de la Ley de Contratos del Sector Público. Boletín Oficial del Estado, $\mathrm{n}^{0}$ 276. España.

(15) Montes, M. V. (2016). Sistema de Clasificación de Procesos Básicos y Sistema de Clasificación de Procesos de Ejecución. ResearchGate, doi: http://dx.doi.org/10.13140/RG.2.1.3517.2883. 